\title{
Ant Colony Optimization for Social Utility Maximization in a Multiuser Communication System
}

\author{
Ming-Hua Lin, ${ }^{1}$ Jung-Fa Tsai, ${ }^{2}$ and Lu-Yao Lee ${ }^{1}$ \\ ${ }^{1}$ Department of Information Technology and Management, Shih Chien University, No. 70, Dazhi Street, Taipei 10462, Taiwan \\ ${ }^{2}$ Department of Business Management, National Taipei University of Technology, No. 1, Section 3, Zhongxiao E. Road, \\ Taipei 10608, Taiwan
}

Correspondence should be addressed to Jung-Fa Tsai; jftsai@ntut.edu.tw

Received 3 May 2013; Accepted 4 July 2013

Academic Editor: Yi-Chung Hu

Copyright (C) 2013 Ming-Hua Lin et al. This is an open access article distributed under the Creative Commons Attribution License, which permits unrestricted use, distribution, and reproduction in any medium, provided the original work is properly cited.

\begin{abstract}
In a multiuser communication system such as cognitive radio or digital subscriber lines, the transmission rate of each user is affected by the channel background noise and the crosstalk interference from other users. This paper presents an efficient ant colony optimization algorithm to allocate each user's limited power on different channels for maximizing social utility (i.e., the sum of all individual utilities). The proposed algorithm adopts an initial solution that allocates more power on the channel with a lower background noise level. Besides, the cooling concept of simulated annealing is integrated into the proposed method to improve the convergence rate during the local search of the ant colony optimization algorithm. A number of experiments are conducted to validate the effectiveness of the proposed algorithm.
\end{abstract}

\section{Introduction}

In a modern communication system such as cognitive radio or digital subscriber lines (DSL), multiple users share the same frequency band and how to mitigate interference is a major design and management concern. The frequency division multiple access (FDMA) mechanism is a standard approach to eliminate interference by dividing the spectrum into multiple tones and preassigning them to the users on a nonoverlapping basis. However, this approach may lead to high system overhead and low bandwidth utilization. Therefore, how to allocate spectrum dynamically to enhance overall system utility in a multiuser communication system has become a topic of intensive research in the signal processing and digital communication community.

From the optimization perspective, the study of the dynamic spectrum management problem can be divided into three kinds of categories $[1,2]$ : noncooperative Nash equilibrium, cooperative social utility maximization, and competitive economy equilibrium. Several approaches were proposed to compute a Nash equilibrium solution such as iterative waterfilling algorithm (IWFA) $[3,4]$ and linear complementarity problem (LCP) [5]. Some algorithms [69] were developed to compute a globally optimal power allocation for the cooperative game. Walras [10] proposed the competitive equilibrium concept which dynamically balances by the supply and demand to model the market operation. Ye [2] proposed a competitive economy equilibrium solution that may achieve both social economic efficiency and individual optimality in dynamic spectrum management. Lin et al. [11] further observed that adjusting budget can meet each user's physical power demand or balance all individual utilities in a competitive spectrum market. Xie et al. [13] proposed a decentralized tâtonnement process for adjusting the prices to achieve a competitive equilibrium. They showed that competitive equilibrium is the solution of a linear complementarity problem and can be computed efficiently.

It is difficult to optimize the overall system utility if only each user's individual utility is improved. Yu et al. [14] proposed a method which refined the optimal spectrum management with less complexity and generalized the multiuser power management for orthogonal frequency division multiplex systems maximized the overall utility. Due to the nonconvexity of this problem, the CPU time required 


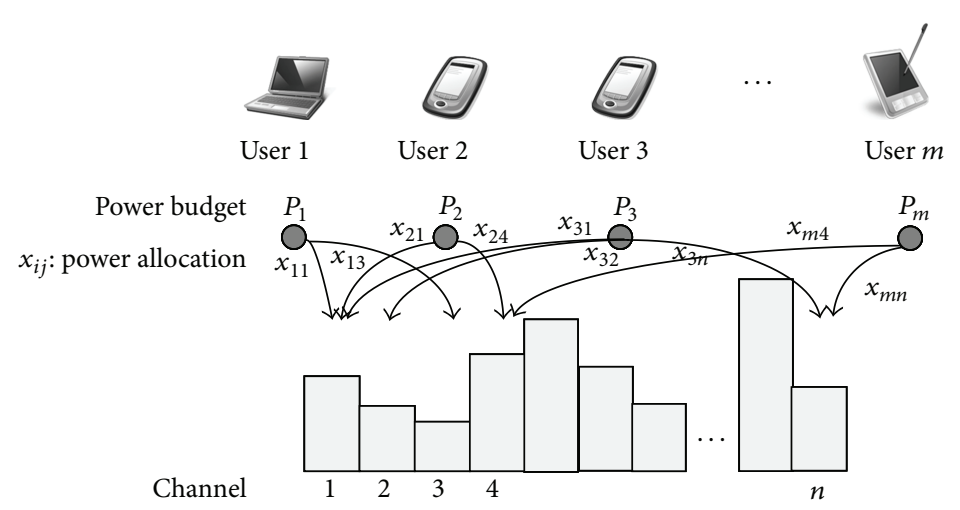

FIGURE 1: The power allocation problem in a multiuser communication system.

to solve this problem by the conventional mathematical programming techniques increases greatly as the number of users and channels increases. Luo and Zhang [1] presented a systematic study of the dynamic spectrum management problem, covering two key theoretical aspects: complexity and duality. They have determined the complexity status of the spectrum management problem under various practical settings as well as different choices of system utility functions and have identified subclasses which are polynomial time solvable. They proved that the sum-rate maximization problem is NP-hard even when there are only two users in the system. Ma et al. [15] proposed an efficient scheduling algorithm for concurrent transmissions with power control to maximize the spectrum utilization and thus the network throughput in broadband wireless networks. The original nonconvex problem is converted into a series of convex problems using a two-step approximation. Then a power and channel allocation with fairness algorithm is proposed to solve these problems. Simulation results showed that both network throughput and fairness are improved by their method compared to the previously developed scheduling algorithms.

Although the heuristic algorithms cannot guarantee global optimality, they have the advantages of robustness and easy implementation for complex problems. In this paper, we propose an efficient ant colony optimization algorithm to allocate each user's limited power on different channels for maximizing social utility (i.e., the sum of all individual utilities). In order to improve the solution efficiency, the proposed algorithm adopts an initial solution that allocates more power on the channel with a lower background noise level. Besides, the cooling concept of simulated annealing is integrated into the proposed method to improve the convergence rate during the local search of the ant colony optimization algorithm. A number of experiments are conducted to validate the effectiveness and contribution of the proposed algorithm. Comparing with the simulated annealing approach, computational results indicate that the proposed algorithm spends less CPU time but finds a solution with a higher social utility. Comparing with mathematical programming solver LINGO, the differences in social utility obtained between the proposed algorithm and LINGO are within $0.5 \%$ for all cases, and the proposed algorithm obviously decreases CPU time for solving large-scale problems.

The rest of this paper is organized as follows. Section 2 gives an overview of the problem formulation of the social utility maximization problem as well as ant colony optimization techniques. Section 3 describes the proposed ant colony optimization algorithm. Section 4 presents the extensive experimental results and analysis of the proposed algorithm. Finally, concluding remarks are made in Section 5.

\section{Background Information}

2.1. Social Utility Maximization Problem. Consider a communication system consisting of $m$ users and $n$ channels indicated in Figure 1. Multiple users may use the same channel at the same time and cause interference to each other. Suppose the power allocated by user $i$ at channel $j$ is $x_{i j}>0$, the total power allocated by user $i$ is bounded by the power budget $P_{i}, \sum_{j=1}^{n} x_{i j} \leq P_{i}$. To evaluate each user's utility, a commonly recognized utility for user $i$ in communication is the Shannon utility $[2,16]$ :

$$
u_{i}\left(\mathbf{x}_{i}, \overline{\mathbf{x}}_{i}\right)=\sum_{j=1}^{n} \log \left(1+\frac{x_{i j}}{\sigma_{i j}+\sum_{k \neq i} a_{k j}^{i} x_{k j}}\right),
$$

where variable $\mathbf{x}_{i}=\left(x_{i 1}, x_{i 2}, \ldots, x_{i n}\right)$ is the power allocation of user $i$ on each channel, variables in $\overline{\mathbf{x}}_{i}=$ $\left(\mathbf{x}_{1}, \ldots, \mathbf{x}_{i-1}, \mathbf{x}_{i+1}, \ldots, \mathbf{x}_{m}\right)$ are the power units allocated by all other users on each channel, parameter $\sigma_{i j}$ denotes the normalized background noise power for user $i$ at channel $j$, and parameter $a_{k j}^{i}$ is the normalized crosstalk ratio from user $k$ to user $i$ at channel $j$. Due to multiuser interference, user $i$ 's utility evaluated by Shannon utility depends on not only its own power allocation on each channel but also those of others. This study aims to maximize the overall system throughput, which is the sum of individual rates in each frequency carrier. Since each user has a fixed transmit power budget $P_{i}$, the social utility maximization problem (sum-rate maximization problem) can be formulated as follows: 
Model 1:

$$
\begin{array}{cl}
\operatorname{maximize} & \sum_{i=1}^{m} \sum_{j=1}^{n} \log \left(1+\frac{x_{i j}}{\sigma_{i j}+\sum_{k \neq i} a_{k j}^{i} x_{k j}}\right) \\
\text { subject to } & \sum_{j=1}^{n} x_{i j} \leq P_{i}, \quad i=1, \ldots, m, \\
x_{i j} \geq 0, \quad i=1, \ldots, m, j=1, \ldots, n,
\end{array}
$$

where $x_{i j}$ are variables and the other are parameters.

Consider a communication system with two channels $\left(f_{1}\right.$ and $f_{2}$ ) and two users, each of them has a physical power constraint $P_{1}=P_{2}=1$. Let the Shannon utility function for user 1 be

$$
\log \left(1+\frac{x_{11}}{1+x_{21}}\right)+\log \left(1+\frac{x_{12}}{4+x_{22}}\right)
$$

and one for user 2 be

$$
\log \left(1+\frac{x_{21}}{2+x_{11}}\right)+\log \left(1+\frac{x_{22}}{4+x_{12}}\right),
$$

and let the aggregate social utility be the sum of the two individual user utilities. The social utility maximization problem for the above system can be formulated as follows.

Model 2:

$$
\begin{array}{cc}
\operatorname{maximize} & \log \left(1+\frac{x_{11}}{1+x_{21}}\right)+\log \left(1+\frac{x_{12}}{4+x_{22}}\right) \\
+\log \left(1+\frac{x_{21}}{2+x_{11}}\right)+\log \left(1+\frac{x_{22}}{4+x_{12}}\right) \\
\text { subject to } \quad x_{11}+x_{12} \leq 1, \quad x_{21}+x_{22} \leq 1 \\
x_{11}, x_{12}, x_{21}, x_{22} \geq 0
\end{array}
$$

The Nash equilibrium of Model 2 is $\left(x_{11}, x_{12}\right)=(1,0)$ and $\left(x_{21}, x_{22}\right)=(1,0)$, where the social utility has value 0.3010 . However, the solution for maximizing social utility is $\left(x_{11}, x_{12}\right)=(1,0)$ and $\left(x_{21}, x_{22}\right)=(0,1)$, where the social utility has value 0.3979 . This paper aims to find the power allocation for each user to achieve the maximal social utility, that is, the highest system throughput. The above sum-rate maximization problem (Model 1) has been proven to be NPhard even when only two users are available in the system. In this paper, we propose an efficient ant colony optimization algorithm to allocate each user's limited power on different channels for maximizing social utility.

2.2. Ant Colony Optimization Techniques. Although a wide variety of deterministic optimization techniques are proposed to solve optimization problems, many of these methods have inherent disadvantages because they require continuous evaluation of derivatives and become prohibitively ill conditioned for large-scale problems [17]. Several heuristic methods inspired from physics, biology, and social science have been developed in recent years such as genetic algorithm, simulated annealing, particle swarm optimization, tabu search, neural nets, and ant colony systems. Ant colony optimization (ACO) which simulates the action of ants was first introduced by Dorigo [18]. ACO aims at mimicking the way almost blind ants establish the shortest route from their nests to the food source and back. It was observed that a moving ant deposits some pheromone on the paths they traverse. New ants joining the search, attracted by the pheromone, further reinforce the path. With time, a series of feasible routes are traversed by teams of ants. As the pheromone evaporates, solutions with no substantial travelers will have difficulty in maintaining their pheromone trails and infeasible routes are gradually eliminated [19]. The complete surveys of applications, models, and algorithmic advances for ACO through 2006 are provided by Blum [20], Cordon et al. [21], Dorigo et al. [22], Dorigo and Stützle [23], and Lim et al. [19].

The ant colony algorithm was originally used for solving combinatorial optimization problems including the traveling salesman problem. Then it has been applied to other optimization problems such as quadratic assignment, vehicle routing, sequential orderings, job shop scheduling, power system optimization, telecommunication network routing, and logic circuit design [17, 19]. Although ACO has been proven to be one of the best metaheuristics in some combinatorial optimization problems, the application to the realparameter optimizations appears more challenging, since the pheromone laying method is not straightforward. The algorithms for continuous function optimization were first developed by Bilchev and Parmee [24]. Attention in these works was devoted only on local search processes. Wodrich and Bilchev [25] extended and modified the earlier algorithm to make it an effective global optimization procedure for continuous problem spaces. Later, several approaches [2631] have been proposed for continuous optimization in recent years. The complete surveys and comparisons of using ACO for continuous cost function optimization can be found in de França et al. [26] and Korošec and Šilc [28].

\section{The Proposed Algorithm}

This study proposes an efficient ant colony optimization algorithm to allocate each user's limited power on different channels for maximizing social utility. To improve the solution efficiency, the proposed algorithm uses an initial solution that allocates more power on the channel with a lower background noise. Moreover, the cooling concept of simulated annealing is incorporated into the proposed method to improve the convergence rate during the local search of the ant colony optimization algorithm. The flowchart of the proposed simulated annealing-based ant colony optimization (SAACO) algorithm is shown in Figure 2.

Let $v$ be the number of ants in the colony; the location of ant $k, k=1, \ldots, v$ at time $t$ represents a solution $X_{k}^{t}=$ $\left\{\mathbf{x}_{k, 1}^{t}, \ldots, \mathbf{x}_{k, m}^{t}\right\}$ of the social utility maximization problem, where $\mathbf{x}_{k, i}^{t}=\left(x_{i 1}^{t}, x_{i 2}^{t}, \ldots, x_{i n}^{t}\right)$ and $x_{i j}^{t}$ is the power units allocated by user $i$ at channel $j$ at time $t$. More detail of the 


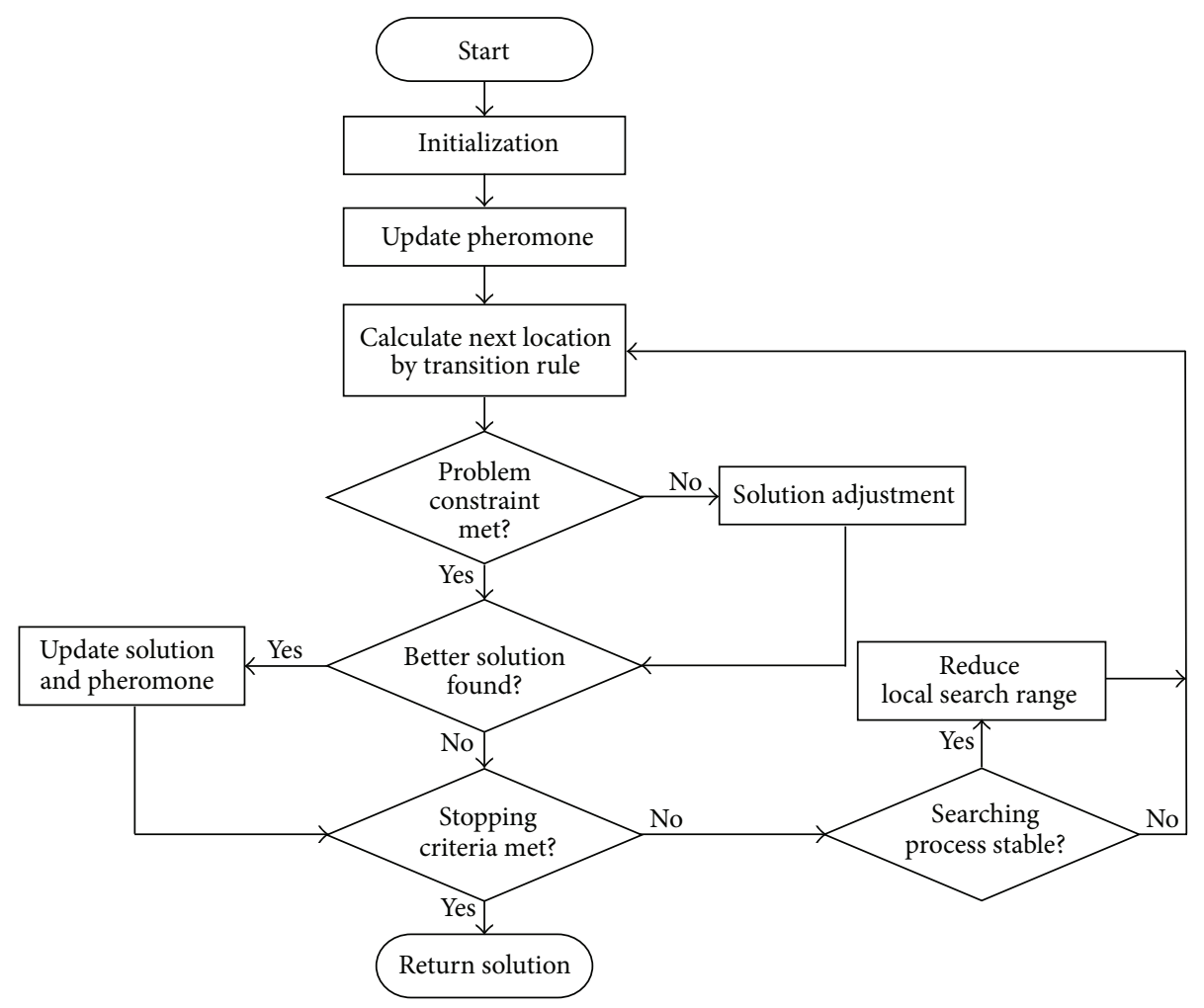

FIGURE 2: Flowchart of the proposed SAACO algorithm.

proposed SAACO algorithm in this study is summarized as follows.

(1) Initialization. $v$ ants are placed in solution space. According to the Shannon utility function, allocating power on the channel with a lower background noise level contributes more to the user utility. The proposed algorithm considers an initial solution based on the background noise level. One half of ants adopt an initial solution considering background noise level, another half generate the initial solution randomly.

(2) Pheromone Updating Rule. Since this study aims to find a solution with maximal social utility (i.e., $\sum_{i=1}^{m} \sum_{j=1}^{n} \log (1+$ $\left.\left.x_{i j} /\left(\sigma_{i j}+\sum_{k \neq i} a_{k j}^{i} x_{k j}\right)\right)\right)$, the pheromone laid on each location is set in proportion to the value of social utility. That is, the pheromone level of visited location by ant $k$ at time $t, \mathrm{ph}_{k}^{t}$, is updated as follows:

$$
\mathrm{ph}_{k}^{t}=(1-p) \mathrm{ph}_{k}^{t-1}+g U\left(X_{k}^{t}\right)
$$

where $p$ is the pheromone evaporation rate, $g$ is a proportional ratio, and $U\left(X_{k}^{t}\right)$ is the social utility of solution $X_{k}^{t}$. The ant deposits more pheromone on a location with a higher social utility value. The pheromone level is updated only when a better solution is found.

(3) State Transition Rule. After objective function (social utility) evaluation, the best solution (i.e., the highest pheromone) location uncovered by the ants is used to guide the search in subsequent generations. Assume that the ant with the highest social utility deposits the most pheromone at time $t$, Best $\mathrm{Ph}^{t}=\max _{k}\left\{\mathrm{ph}_{k}^{t}\right\}$, on its location, then each ant $k$ on the location with its pheromone level $\mathrm{Ph}_{k}^{t}$ uses the following state transition rule to determine the next move:

$$
X_{k}^{t+1}= \begin{cases}X_{k}^{t}+\text { min_step } * d, & \text { if } w \leq G_{0}, \text { (exploitation) } \\ X_{k}^{t}+\text { max_step } * d, & \text { if } w>G_{0}, \text { (exploration) }\end{cases}
$$

where $w=\left(\right.$ Best $\left.\mathrm{Ph}^{t}-\mathrm{Ph}_{k}^{t}\right) /$ Best $\mathrm{Ph}^{t}, d$ is a random variable between 0 and 0.5 , and min_step, max_step, and $G_{0}$ are prespecified constants. To facilitate both exploitation and exploration in the proposed method, an ant searches locally when $w$ is found to be lower than or equal to the user-specific threshold $G_{0}$ and searches globally when $w$ is greater than $G_{0}$.

(4) Solution Adjustment. After an ant uses the transition rule to move next, the solution on next location is verified so that it meets the problem constraint $\left(\sum_{j=1}^{n} x_{i j} \leq P_{i}\right)$. 


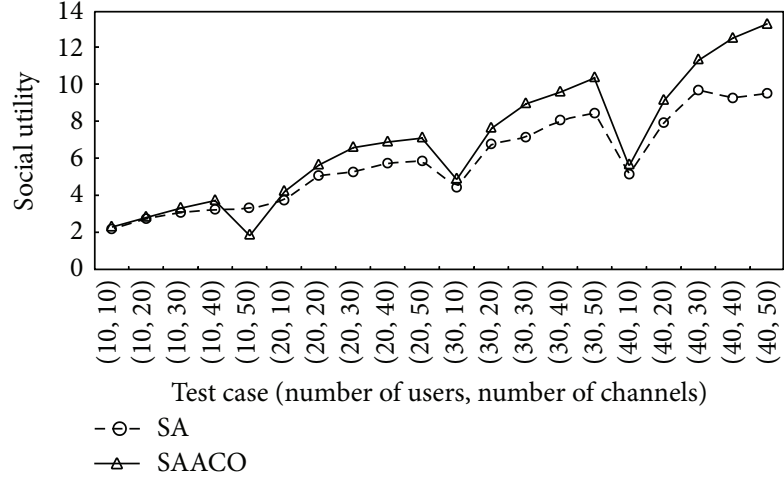

Figure 3: Comparison of social utility by SAACO and SA Lin et al. [12].

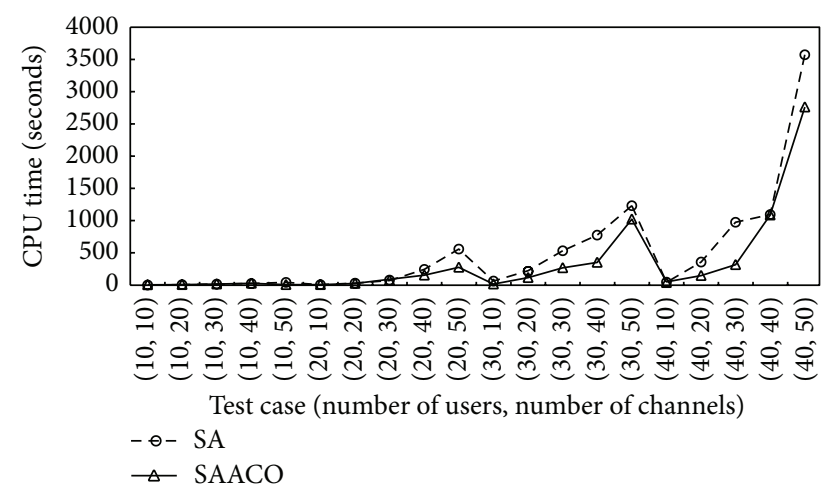

FIgURE 4: Comparison of CPU time by SAACO and SA Lin et al. [12].

If the problem constraint is violated, the proposed method performs solution adjustment to make the next solution a feasible solution.

(i) If $x_{i j}$ is not in the domain $\left(\underline{x}_{i j}, \bar{x}_{i j}\right)$, where $\underline{x}_{i j}$ and $\bar{x}_{i j}$ are lower bound and upper bound of $x_{i j}$, then adjust $x_{i j}$ in its range by the following rule:

$$
x_{i j}= \begin{cases}\bar{x}_{i j}, & \text { if } x_{i j}>\bar{x}_{i j} \\ \underline{x}_{i j}, & \text { if } x_{i j}<\underline{x}_{i j} \\ x_{i j}, & \text { otherwise }\end{cases}
$$

(ii) If $\sum_{j=1}^{n} x_{i j}>P_{i}$, then randomly select one of the following three conditions for adjusting $x_{i j}$.

(a) $x_{i j}$ are adjusted proportionally such that the total power allocation does not exceed its limitation $P_{i}$, that is, $x_{i j}=\left(x_{i j} / \sum_{j=1}^{n} x_{i j}\right) P_{i}$.

(b) Remain the power allocation for the channel with maximal power allocation, and allocate $P_{i}-$ $\max _{j}\left\{x_{i j}\right\}$ on a randomly selected channel.

(c) Allocate all power on the channel with the lowest background noise level.
TABLE 1: Parameter setting for the proposed SAACO algorithm.

\begin{tabular}{llc}
\hline Parameter & Descriptions & Settings \\
\hline$v$ & Number of ants & 10 \\
$R_{\text {step }}$ & Rate to reduce the local search range & 0.9 \\
$S q$ & $\begin{array}{l}\text { Threshold of the local search range for } \\
\text { stopping criteria }\end{array}$ & 0.01 \\
min_step & Local search range & 0.5 \\
max_step & Global search range & 1.0 \\
$p$ & The pheromone evaporation rate & 0.9 \\
$g$ & Proportional ratio & 0.1 \\
$G_{0}$ & Probability of global search & 0.7 \\
\hline
\end{tabular}

(iii) If $\sum_{j=1}^{n} x_{i j}<P_{i}$, then allocate unused power $P_{i}-$ $\sum_{j=1}^{n} x_{i j}$ to the channel that gets the lowest power allocated.

(5) Local Search Range Reduction. If there is no improvement in solution quality after a user-defined number of iterations in the searching process, the searching process is stable and the proposed method incorporates the concept of simulated annealing to reduce the local search range from min_step to min_step $* R_{\text {step }}$, where $R_{\text {step }}$ is between 0.8 and 0.99 .

(6) Stopping Criteria. After min_step is below the userspecific threshold $S q$, terminate the searching process.

\section{Experimental Results and Discussions}

In this section, the effectiveness of the proposed SAACO method is validated using experimental data. All experiments are implemented using $\mathrm{C}++$ language under the circumstance of Windows XP on an Intel CPU P4 1.7 G computer with $1 \mathrm{~GB}$ memory. In all simulated cases, each user's power constraint $P_{i}=1$, the channel background noise level $\sigma_{i j}$ are chosen randomly from the interval $[1,9]$, and the normalized crosstalk ratio $a_{k j}^{i}$ are chosen randomly from the interval $[0.1,0.9]$.

4.1. Parameters Settings. Since different parameter settings significantly affect the performance of the proposed SAACO algorithm, a number of experiments are run to determine suitable parameter settings for the algorithm listed in Table 1.

4.2. Comparison between SAACO and SA. We compare the proposed SAACO approach with the simulated annealing (SA) approach proposed by Lin et al. [12] in the case of $m$ users $(m=10,20,30,40)$ and $n$ channels $(n=$ $10,20,30,40,50)$. Figures 3 and 4 compare social utility and CPU time, respectively, by SAACO and SA. We find that the proposed SAACO algorithm spends less CPU time but obtains better solutions with higher social utilities than the SA approach Lin et al. [12]. 
TABLE 2: Comparisons of CPU time and social utility between the proposed SAACO approach and LINGO.

\begin{tabular}{|c|c|c|c|c|c|c|}
\hline \multirow{2}{*}{ Test case $(m, n)^{*}$} & \multicolumn{2}{|c|}{ LINGO } & \multicolumn{2}{|c|}{ SAACO } & \multicolumn{2}{|c|}{ Difference } \\
\hline & Social utility & CPU time (seconds) & Social utility & CPU time (seconds) & Difference_s ${ }^{+}$ & Difference $\_t^{++}$ \\
\hline$(10,10)$ & 2.422536 & 1.0 & 2.418003 & 1.214600 & -0.001871 & 0.214600 \\
\hline$(10,20)$ & 3.027710 & 4.0 & 3.029383 & 4.901600 & 0.000553 & 0.225400 \\
\hline$(10,30)$ & 3.454207 & 10.5 & 3.448223 & 15.805900 & -0.001732 & 0.505324 \\
\hline$(10,40)$ & 3.665038 & 21.2 & 3.660824 & 22.857000 & -0.001150 & 0.078160 \\
\hline$(10,50)$ & 3.737554 & 31.3 & 3.7343727 & 36.64825 & -0.000851 & 0.171994 \\
\hline$(20,10)$ & 3.981162 & 5.6 & 3.998787 & 7.241300 & 0.004427 & 0.293089 \\
\hline$(20,20)$ & 5.575991 & 30.9 & 5.60343 & 26.974900 & 0.004921 & -0.127026 \\
\hline$(20,30)$ & 6.447189 & 88.9 & 6.440597 & 79.355200 & -0.001023 & -0.107366 \\
\hline$(20,40)$ & 6.942952 & 215.7 & 6.943312 & 190.842300 & 0.000052 & -0.115242 \\
\hline$(20,50)$ & 7.265195 & 375.3 & 7.250848 & 347.890200 & -0.001975 & -0.073034 \\
\hline
\end{tabular}

${ }^{*}(m, n)=$ (number of users, number of channels).

${ }^{+}$Difference_s $=($social utility by SAACO - social utility by LINGO $) /$social utility by LINGO.

${ }^{++}$Difference_t $=($CPU time by SAACO - CPU time by LINGO $) /$CPU time by LINGO.

TABLE 3: Comparisons of CPU time and social utility between the proposed SAACO approach and LINGO in large scale problem.

\begin{tabular}{|c|c|c|c|c|c|c|}
\hline \multirow{2}{*}{ Test case $(m, n)^{*}$} & \multicolumn{2}{|c|}{ LINGO } & \multicolumn{2}{|c|}{ SAACO } & \multicolumn{2}{|c|}{ Differrence } \\
\hline & Sociall utility & CPU time (seconds) & Sociall utility & CPU time (seconds) & Difference_s ${ }^{+}$ & Difference_t $\mathrm{t}^{++}$ \\
\hline$(10,100)$ & 4.064655 & 238.333 & 4.054523 & 238.439 & -0.00249 & 0.00044 \\
\hline$(10,128)$ & 4.122419 & 563.667 & 4.107250 & 362.911 & -0.00368 & -0.55318 \\
\hline$(10,256)$ & 4.162954 & 3906.667 & 4.217743 & 2835.007 & 0.01316 & -0.37801 \\
\hline$(20,100)$ & 7.911308 & 3224.000 & 7.888093 & 1608.873 & -0.00293 & -1.00389 \\
\hline$(20,128)$ & 8.061679 & 4807.000 & 8.031333 & 3144.747 & -0.00376 & -0.52858 \\
\hline$(20,256)$ & 8.372676 & 45587.000 & 8.354917 & 21984.100 & -0.00212 & -1.07364 \\
\hline
\end{tabular}

${ }^{*}(m, n)=$ (number of users, number of channels).

${ }^{+}$Difference_s $=($social utility by SAACO - social utility by LINGO) $/$social utility by LINGO.

${ }^{++}$Difference_t $=($CPU time by SAACO - CPU time by LINGO $) /$CPU time by LINGO.

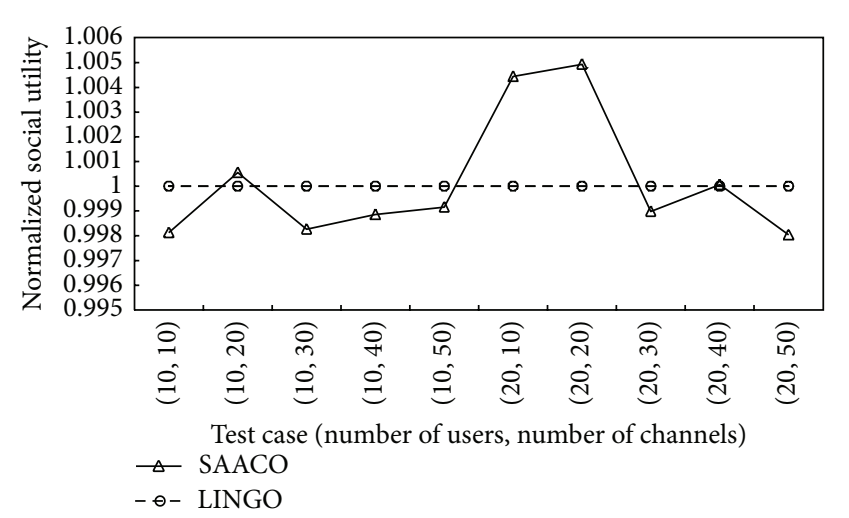

FIGURE 5: Comparison of social utility by SAACO and LINGO.

4.3. Comparison between SAACO and LINGO. In this section, some experiments are conducted to compare the proposed SAACO approach with the optimization software LINGO [32]. First we consider small-scale problems of $m$ users $(m=10,20)$ and $n$ channels $(n=10,20,30,40,50)$. Ten problems are randomly generated for each group. Table 2 compares average performance of the proposed SAACO

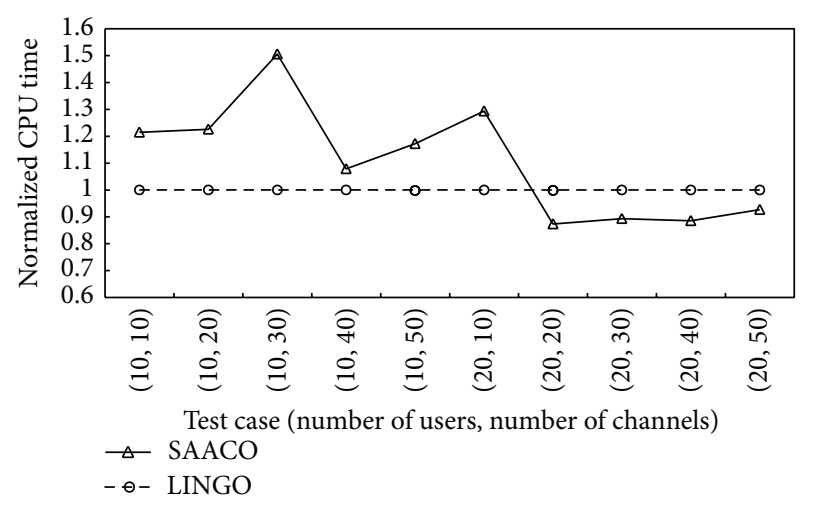

FIGURE 6: Comparison of CPU time by SAACO and LINGO. approach and LINGO. To compare SAACO and LINGO more clearly, we normalize the data and plot it in Figures 5 and 6. In Figure 5, we observe that the difference between social utility by LINGO and by SAACO is between $+0.5 \%$ and $-0.2 \%$. Besides, the proposed SAACO approach uses less CPU time than LINGO in obtaining an optimal solution when 20 users and over 20 channels exist in the communication system. 


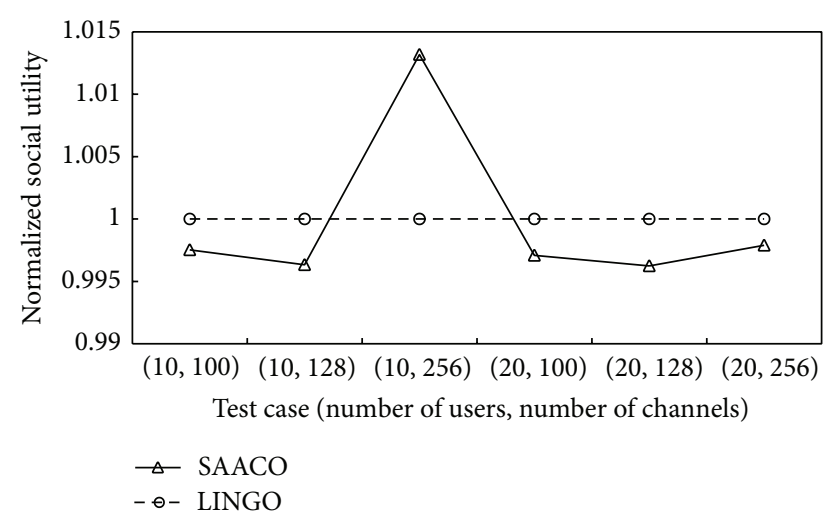

FIGURE 7: Comparison of social utility by SAACO and LINGO in large-scale problems.

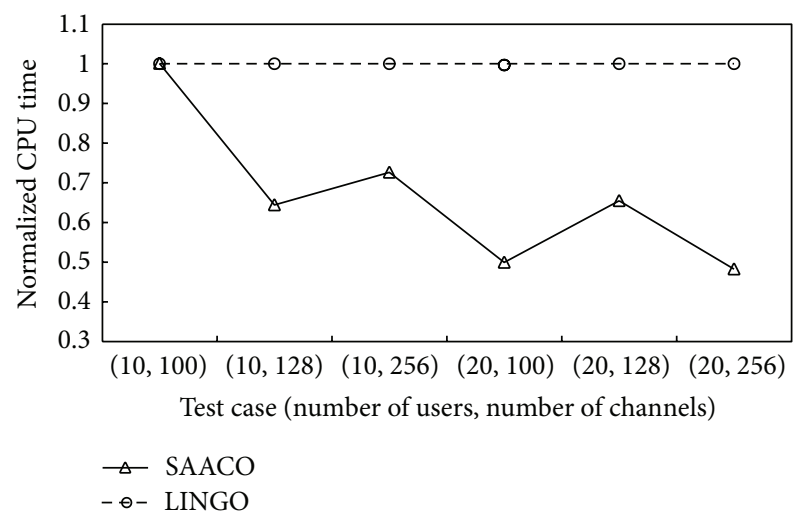

FIGURE 8: Comparison of CPU time by SAACO and LINGO in large-scale problems.

To demonstrate effectiveness of the proposed method in large-scale problems, we compare the proposed SAACO approach with LINGO in the cases of $m$ users $(m=10,20)$ and $n$ channels $(n=100,128,256)$. Three problems are randomly generated for each group. Table 3 compares average performance of SAACO and LINGO measured by the resulting social utility and the required CPU time. To compare SAACO and LINGO more clearly, we normalize the data and plot it in Figures 7 and 8. Figure 8 indicates that the proposed SAACO algorithm performs better than LINGO in CPU time as the number of users and channels increases. Compared with LINGO, the proposed SAACO approach uses roughly $50 \%$ of CPU time to obtain the solution in the case of 20 users and 256 channels. Although the required CPU time by the proposed SAACO approach is much less than LINGO, the proposed SAACO approach can get a better social utility or an approximate social utility not less than 1 percent from the solution reported by LINGO as indicated in Figure 7.

\section{Conclusions}

With the rapid development of wireless networks and devices, the demand for spectrum resource is increasingly growing. Therefore, how to allocate spectrum dynamically to enhance total system throughput in a multiuser communication system has become an important issue in recent years. Due to the nonconvexity of this problem, the CPU time required to solve this problem by the conventional mathematical programming techniques increases greatly as the number of channel and user increases. This paper proposes an efficient ant colony algorithm for allocating spectrum to maximize social utility. Computational results show that the proposed algorithm significantly outperforms the simulated annealing approach in solution efficiency and solution quality in most cases. Comparing with the mathematical programming solver LINGO in solving large-scale problems, the proposed SAACO algorithm spends much less CPU time obtaining an approximate social utility close to the social utility reported from LINGO.

\section{Acknowledgment}

The research is supported by Taiwan NSC Grants NSC 1012410-H-158-002-MY2 and NSC 102-2410-H-027-012-MY3.

\section{References}

[1] Z.-Q. Luo and S. Zhang, "Dynamic spectrum management: complexity and duality," IEEE Journal on Selected Topics in Signal Processing, vol. 2, no. 1, pp. 57-73, 2008.

[2] Y. Ye, "Competitive communication spectrum economy and equilibrium," 2007, http://www.stanford.edu/ yyye/spectrum pricing3.pdf.

[3] S. T. Chung, S. J. Kim, J. Lee, and J. M. Cioffi, "A game-theoretic approach to power allocation in frequency-selective Gaussian interference channels," in Proceedings of IEEE International Symposium on Information Theory (ISIT '03), p. 316, Kanagawa, Japan, July 2003.

[4] W. Yu, G. Ginis, and J. M. Cioffi, "Distributed multiuser power control for digital subscriber lines," IEEE Journal on Selected Areas in Communications, vol. 20, no. 5, pp. 1105-1115, 2002.

[5] Z.-Q. Luo and J.-S. Pang, "Analysis of iterative waterfilling algorithm for multiuser power control in digital subscriber lines," EURASIP Journal on Applied Signal Processing, vol. 2006, Article ID 024012, 10 pages, 2006.

[6] R. Cendrillon, J. Huang, M. Chiang, and M. Moonen, "Autonomous spectrum balancing for digital subscriber lines," IEEE Transactions on Signal Processing, vol. 55, no. 8, pp. 42414257, 2007.

[7] V. M. K. Chan and W. Yu, "Joint multiuser detection and optimal spectrum balancing for digital subscriber lines," EURASIP Journal on Applied Signal Processing, vol. 2006, Article ID 080941, 13 pages, 2006.

[8] R. Lui and W. Yu, "Low-complexity near-optimal spectrum balancing for digital subscriber lines," in Proceedings of IEEE International Conference on Communications (ICC '05), pp. 1947-1951, Seoul, Korea, May 2005.

[9] W. Yu and R. Lui, "Dual methods for nonconvex spectrum optimization of multicarrier systems," IEEE Transactions on Communications, vol. 54, no. 7, pp. 1310-1322, 2006.

[10] L. Walras, Elements of Pure Economics; or the Theory of Social Wealth, Porcupine Press, Paris, France, 1874.

[11] M.-H. Lin, J.-F. Tsai, and Y. Ye, "Budget allocation in a competitive communication spectrum economy," EURASIP Journal on 
Advances in Signal Processing, vol. 2009, Article ID 963717, 12 pages, 2009.

[12] Y.-C. Lin, M.-H. Lin, H.-H. Huang, and L.-Y. Lee, "Using simulated annealing algorithm for maximizing social utility in dynamic spectrum management," WSEAS Transactions on Communications, vol. 8, no. 7, pp. 638-647, 2009.

[13] Y. Xie, B. Armbruster, and Y. Ye, "Dynamic spectrum management with the competitive market model," IEEE Transactions on Signal Processing, vol. 58, no. 4, pp. 2442-2446, 2010.

[14] W. Yu, R. Lui, and R. Cendrillon, "Dual optimization methods for multiuser orthogonal frequency division multiplex systems," in IEEE Global Telecommunications Conference (GLOBECOM '04), pp. 225-229, Dallas, Tex, USA, December 2004.

[15] B. Ma, Z. Yang, L. Cai, and T. A. Gulliver, "Power allocation and scheduling for broadband wireless networks considering mutual interference," in Proceedings of IEEE International Conference on Communications (ICC '11), pp. 1-5, Kyoto, Japan, June 2011.

[16] T. M. Cover and J. A. Thomas, Elements of Information Theory, John Wiley \& Sons, New York, NY, USA, 1991.

[17] M. Mathur, S. B. Karale, S. Priye, V. K. Jayaraman, and B. D. Kulkarni, "Ant colony approach to continuous function optimization," Industrial and Engineering Chemistry Research, vol. 39, no. 10, pp. 3814-3822, 2000.

[18] M. Dorigo, Optimization, learning and natural algorithms [Ph.D. dissertation], Politecnico di Milano, Milano, Italy, 1992.

[19] K. K. Lim, Y.-S. Ong, M. H. Lim, X. Chen, and A. Agarwal, "Hybrid ant colony algorithms for path planning in sparse graphs," Soft Computing, vol. 12, no. 10, pp. 981-994, 2008.

[20] C. Blum, "Ant colony optimization: introduction and recent trends," Physics of Life Reviews, vol. 2, no. 4, pp. 353-373, 2005.

[21] O. Cordon, F. Herrera, and T. Stützle, "A review on the ant colony optimization metaheuristic: basis, models and new trends," Mathware and Soft Computing, vol. 9, no. 2-3, pp. 141$175,2002$.

[22] M. Dorigo, M. Birattari, and T. Stützle, "Ant colony optimization artificial ants as a computational intelligence technique," IEEE Computational Intelligence Magazine, vol. 1, no. 4, pp. 28-39, 2006.

[23] M. Dorigo and T. Stützle, Ant Colony Optimization, MIT Press, Cambridge, UK, 2004.

[24] G. Bilchev and I. C. Parmee, "The ant colony metaphor for searching continuous design spaces," in Evolutionary Computing, vol. 993 of Lecture Notes in Computer Science, pp. 25-39, Springer, 1995.

[25] M. Wodrich and G. Bilchev, "Cooperative distributed search: the ants' way," Control and Cybernetics, vol. 26, no. 3, pp. 413441, 1997.

[26] F. O. de França, G. P. Coelho, F. J. von Zuben, and R. R. de Faissol Attux, "Multivariate ant colony optimization in continuous search spaces," in Proceedings of the 10th Annual Genetic and Evolutionary Computation Conference (GECCO '08), pp. 9-16, New York, NY, USA, July 2008.

[27] J. Dréo and P. Siarry, "A new ant colony algorithm using the heterarchical concept aimed at optimization of multiminima continuous functions," in Ant Algorithms, vol. 2463 of Lecture Notes in Computer Science, pp. 216-221, Springer, 2002.

[28] P. Korošec and J. Šilc, "A stigmergy-based algorithm for continuous optimization tested on real-life-like environment," in Applications of Evolutionary Computing, vol. 5484 of Lecture Notes in Computer Science, pp. 675-684, Springer, 2009.
[29] N. Monmarché, G. Venturini, and M. Slimane, "On how Pachycondyla apicalis ants suggest a new search algorithm," Future Generation Computer Systems, vol. 16, no. 8, pp. 937-946, 2000.

[30] S. H. Pourtakdoust and H. Nobahari, "An extension of ant colony system to continuous optimization problems," in Ant Colony Optimization and Swarm Intelligence, vol. 3172 of Lecture Notes in Computer Science, pp. 294-301, Springer, 2004.

[31] K. Socha and M. Dorigo, "Ant colony optimization for continuous domains," European Journal of Operational Research, vol. 185, no. 3, pp. 1155-1173, 2008.

[32] Lindo, LINGO Release 8.0, LINDO System Inc., Chicago, Ill, USA, 2004. 


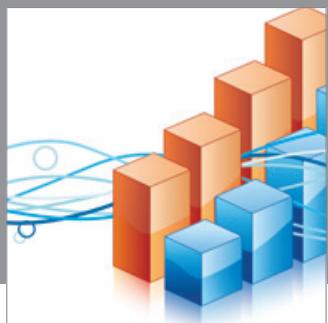

Advances in

Operations Research

mansans

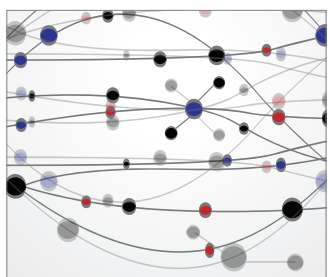

The Scientific World Journal
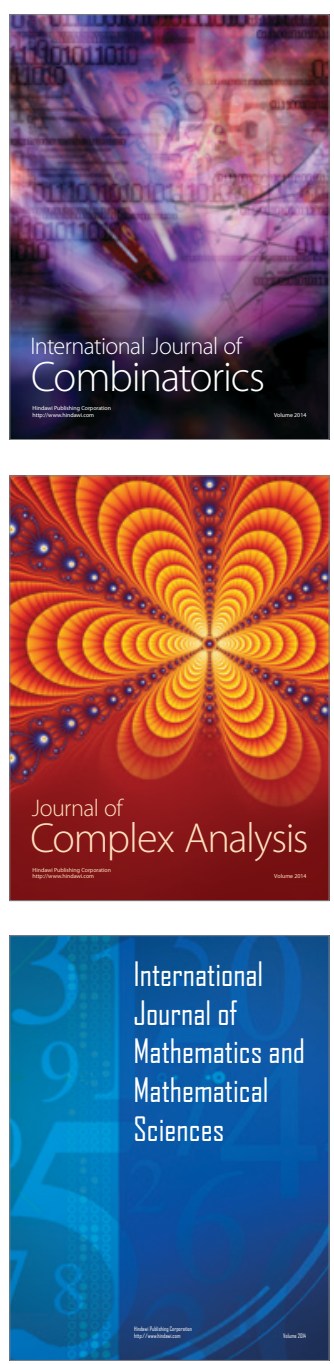
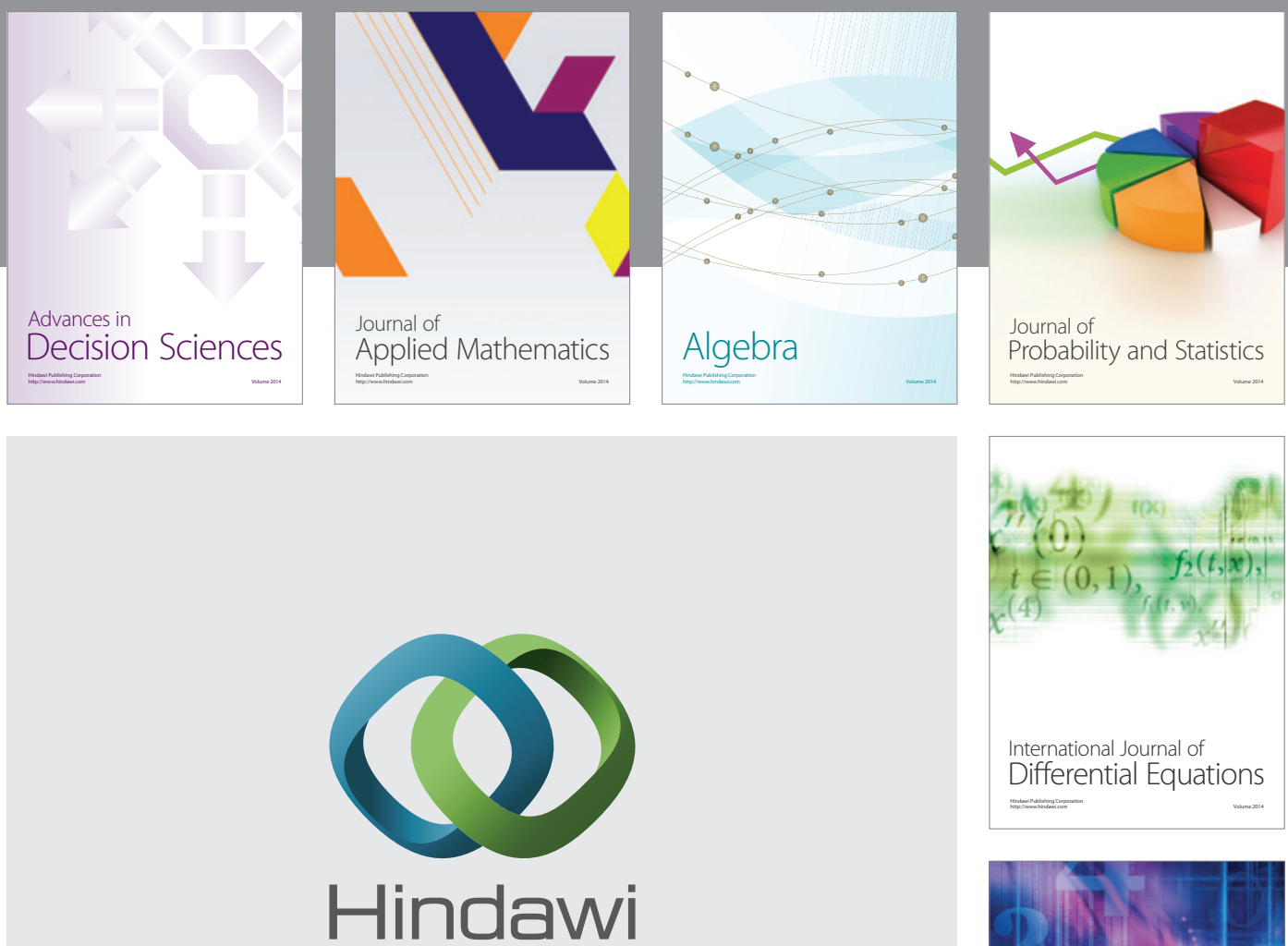

Submit your manuscripts at http://www.hindawi.com
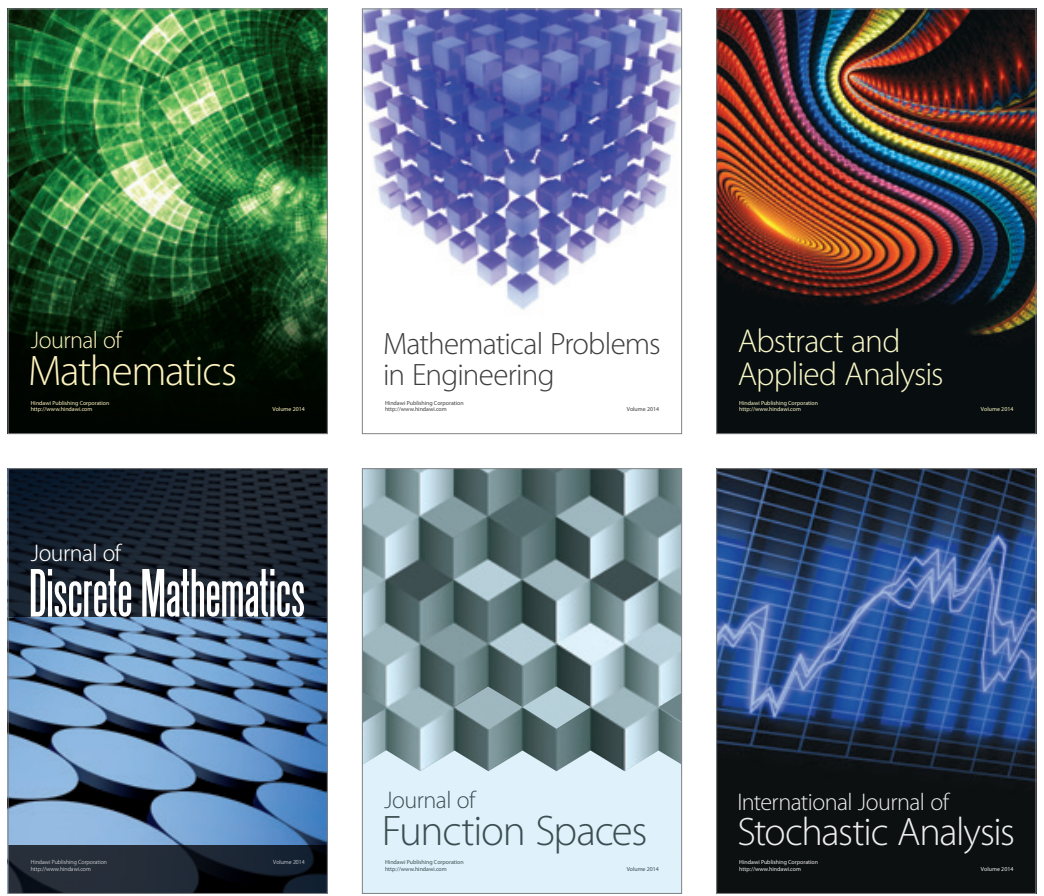

Journal of

Function Spaces

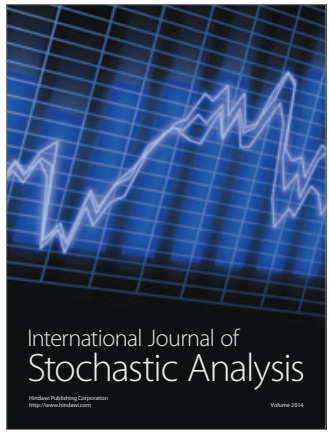

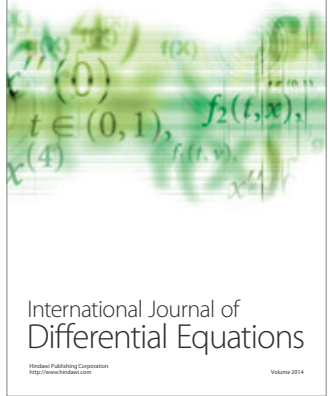
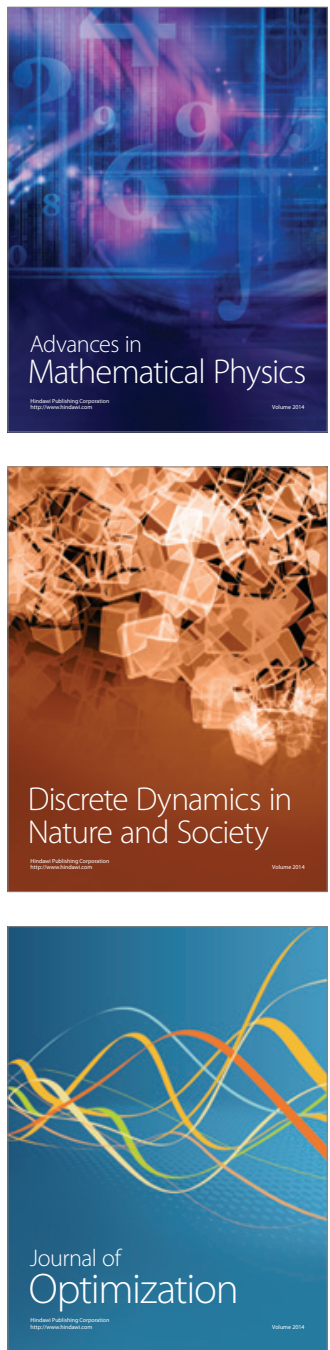